\title{
Magnetic and Related Properties of Ternary TmTX Intermetallics
}

\author{
D. KACZOROWSKI ${ }^{a, *}$ AND A. SzYTUŁA ${ }^{b}$ \\ ${ }^{a}$ Institute of Low Temperature and Structure Research, Polish Academy of Sciences, \\ P.O. Box 1410, 50-950 Wrocław, Poland \\ ${ }^{b}$ M. Smoluchowski Institute of Physics, Jagiellonian University, W.S. Reymonta 4, 30-059 Kraków, Poland \\ We report on the magnetic and thermal properties of a few TmTX compounds, where $\mathrm{T}=d$-electron metal \\ and $\mathrm{X}=p$-electron element. In all these ternaries but TmRuGe the thulium magnetic moments order antiferro- \\ or ferromagnetically at low temperatures. The specific heat data confirms the magnetic orderings and reveals the \\ influence of crystalline electric field effects.
}

DOI: 10.12693 /APhysPolA.127.620

PACS: 75.30.-m, 75.30.Cr, 75.30.Kz, 75.50.Ee, 75.47.Np, 75.40.Cx

\section{Introduction}

Since many years, rare earth intermetallics $\mathrm{R}-\mathrm{T}-\mathrm{X}$, where $\mathrm{R}=$ rare earth, $\mathrm{T}=d$-electron and $\mathrm{X}=p$-electron elements, have been a subject of extensive studies due to their intriguing magnetic and electrical properties [1, 2]. Amidst plentitude of such phases with various chemical compositions and stoichiometries, a series of equiatomic compounds RTX received special attention due to simplicity of their crystal structure and large span in their physical behaviors [3]. While much information is available in the literature for the RTX intermetallics with $\mathrm{R}=\mathrm{Ce}-\mathrm{Er}$, relatively little is known on their counterparts with $\mathrm{R}=\mathrm{Tm}$. Most recently, we reported on the magnetic properties of TmRhGe and TmRhGa, which were found to order antiferromagnetically below $6.0 \mathrm{~K} \mathrm{[4]}$ and $3.9 \mathrm{~K}$ [5], respectively. Similarly, we established before antiferromagnetic ordering in TmNiIn, TmPtIn and TmAgGe to set at $T_{\mathrm{N}}=2.5[6], 3.4[7]$ and $4.2 \mathrm{~K}[8]$, respectively.

Here, we give the first account on our on-going studies on a few other Tm-based equiatomic materials, namely TmPdIn, TmAgSn, TmAuGe and TmRuGe. The experimental results are compared with the data previously obtained for the compounds TmNiIn, TmPtIn and TmAgGe.

\section{Experimental details}

Polycrystalline samples of TmTIn $(\mathrm{T}=\mathrm{Ni}, \mathrm{Pd}, \mathrm{Pt})$, $\mathrm{TmAgX}(\mathrm{X}=\mathrm{Sn}, \mathrm{Ge}), \mathrm{TmAuGe}$ and TmRuGe were synthesized by arc melting the stoichiometric amounts of the constituent elements of high purity ( $3 \mathrm{~N}$ for Tm and $4 \mathrm{~N}$ for the other elements) in titanium-gettered argon atmosphere. Subsequently, the buttons were annealed in an evacuated quartz tube at 873 or $1073 \mathrm{~K}$ for one week.

${ }^{*}$ corresponding author; e-mail: D.Kaczorowski@int.pan.wroc.pl
Quality of the products was checked by X-ray powder diffraction (XRD) performed at room temperature using a powder diffractometer XPERT-PRO PANalytical with $\mathrm{Cu} K_{\alpha}$ radiation. The XRD data were analyzed using the Rietveld-type refinement program FullProf [9].

Magnetic measurements were carried out in the temperature range $1.72-300 \mathrm{~K}$ in magnetic fields up to $5 \mathrm{~T}$ using a Quantum Design MPMS-5 SQUID magnetometer. Heat capacity studies were made over the interval 0.35-300 K employing a Quantum Design PPMS-9 experimental platform.

\section{Results and discussion}

The obtained XRD patterns of $\operatorname{TmTX}(\mathrm{T}=\mathrm{Ni}$, $\mathrm{Pd}, \mathrm{Pt})$ and $\mathrm{TmAgX}(\mathrm{X}=\mathrm{Ge}, \mathrm{Sn})$ were easily indexed assuming a hexagonal crystal structure of the ZrNiAl-type (space group $P \overline{6} 2 m$ ). In the case of TmAuGe the hexagonal LiGaGe-type unit cell (space group $P 6_{3} m c$ ) was established, while for TmRuGe an orthorhombic structure of the TiNiSi-type (space group Pnma) was found.

The magnetic data for all the investigated compounds are summarized in Table I. At high temperatures, the magnetic susceptibility obeys the Curie-Weiss law with the effective magnetic moment close to the free $\mathrm{Tm}^{3+}$ ion value $\left(7.52 \mu_{\mathrm{B}}\right)$ and the paramagnetic Curie temperature either attaining a small positive value or being nearly zero kelvin. As an example, the temperature variation of the inverse magnetic susceptibility of TmPdIn is presented in Fig. 1. As can be inferred from the insets to this figure, the compound orders antiferromagnetically at $T_{\mathrm{N}}=2.8 \mathrm{~K}$, and undergoes a metamagneticlike phase transition in an external magnetic field of about 0.5 T. Similar results were found for antiferromagnetic TmAgSn, and previously reported antiferromagnets TmNiIn [6], TmPtIn [7] and TmAgGe [8]. For all these compounds the magnetic moment measured at $1.72 \mathrm{~K}$ in a field of $5 \mathrm{~T}$ is distinctly smaller that the free $\mathrm{Tm}^{3+}$ ion value $\left(7.0 \mu_{\mathrm{B}}\right)$, hence manifesting strong crystalline electric field interactions. In contrast to the above mentioned 


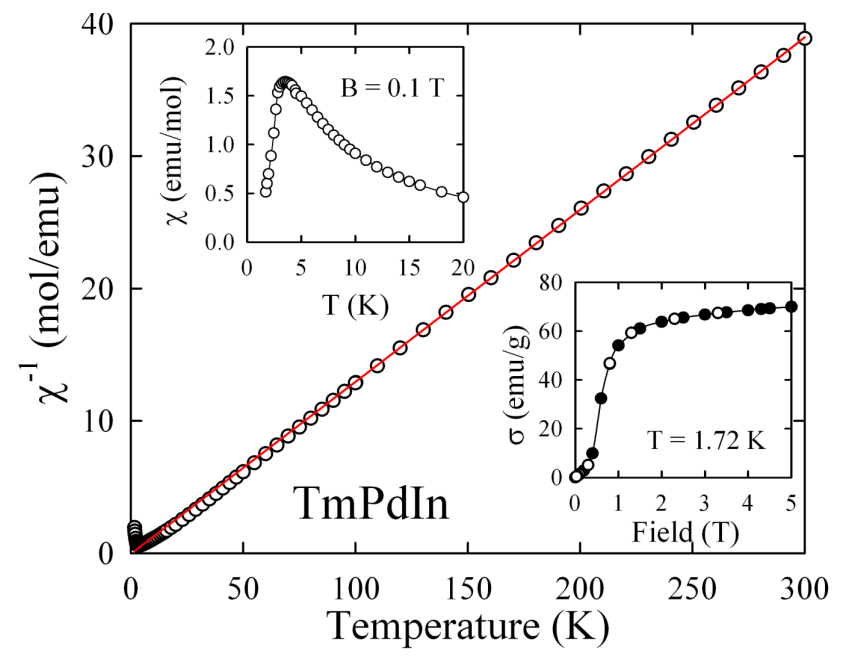

Fig. 1. Temperature dependence of the inverse magnetic susceptibility of TmPdIn. Solid line represents the Curie-Weiss fit. Upper inset: low-temperature $\chi(T)$ data; lower inset: field variation of the magnetization measured at $1.72 \mathrm{~K}$ with increasing and decreasing magnetic field (closed and open symbols, respectively).

ternaries, TmAuGe was found to order ferromagnetically below $T_{\mathrm{C}}=4.1 \mathrm{~K}$, while for $\mathrm{TmRuGe}$ no magnetic phase transition was detected above $1.72 \mathrm{~K}$. Detailed report on the magnetic behavior in these two compounds will be published elsewhere.

TABLE I

Magnetic data of investigated TmTX compounds. $T_{\mathrm{N} / \mathrm{C}}$ - ordering temperature, $\theta_{\mathrm{p}}$ - paramagnetic Curie temperature, $\mu_{\text {eff }}-$ effective magnetic moment, $\mu-$ magnetic moment at $\mathrm{B}=5 \mathrm{~T}$ and $\mathrm{T}=1.72 \mathrm{~K}, \mathrm{H}_{\mathrm{cr}}-$ value of the critical magnetic field.

\begin{tabular}{c|c|c|c|c|c|c}
\hline \hline Compound & $T_{\mathrm{N} / \mathrm{C}}[\mathrm{K}]$ & $\theta_{\mathrm{p}}[\mathrm{K}]$ & $\mu_{\mathrm{eff}}\left[\mu_{\mathrm{B}}\right]$ & $\mu\left[\mu_{\mathrm{B}}\right]$ & $H_{\mathrm{cr}}[\mathrm{T}]$ & Ref. \\
\hline TmNiIn & 2.5 & 0.4 & 7.50 & 4.2 & 0.4 & {$[6]$} \\
TmPdIn & 2.65 & $\approx 0$ & 7.88 & 4.9 & 0.4 & - \\
TmPtIn & 3.4 & 6.5 & 7.65 & 4.5 & 0.5 & {$[7]$} \\
TmAgGe & 4.2 & 12.1 & 7.37 & 3.9 & 0.3 & {$[8]$} \\
TmAgSn & 4.15 & $\approx 0$ & 7.49 & 3.8 & 0.5 & - \\
TmAuGe & 4.1 & 4.0 & 7.53 & 3.9 & - & - \\
TmRuGe & - & $\approx 0$ & 7.52 & 3.4 & - & -
\end{tabular}

The low-temperature dependence of the specific heat of TmPdIn is shown in Fig. 2. It shows a typical $\lambda$ shaped anomaly at $T_{\mathrm{N}}=2.65 \mathrm{~K}$. Additionally, a clear hump in $C(T)$ is seen below $2 \mathrm{~K}$ that can manifest a subsequent order-order phase transition but it can also occur due to Schottky-type contribution to the heat capacity. Further investigations are necessary, preferably using neutron scattering, to conclude on these two scenarios. It should be noted, however, that the entropy released by $T_{\mathrm{N}}$ is only $3.7 \mathrm{~J} /(\mathrm{mol} \mathrm{K})$, i.e. a fraction of the value $R \ln 2=5.7 \mathrm{~J} /(\mathrm{mol} \mathrm{K})$ expected with the molecular field approximation for a doubly-degenerated ground state. Moreover, the observed jump in $C(T)$ at
$T_{\mathrm{N}}$ is about $6 \mathrm{~J} /(\mathrm{mol} \mathrm{K})$ that is only half of the value of $12.47 \mathrm{~J} /(\mathrm{mol} \mathrm{K})$ appropriate for the effective spin $S=1 / 2$. These findings seem to indicate that the electronic ground state in TmPdIn is a singlet that originates from the ${ }^{3} \mathrm{H}_{6}$ multiplet of $\mathrm{Tm}^{3+}$ ion split in an orthorhombic $C_{2 v}$ crystalline electric field (CEF) potential. Hence, the magnetic ordering in the compound possibly has an induced nature and the low-temperature anomaly in $C(T)$ likely arises from the CEF effect. Similar case was reported for TmNiAl [10].

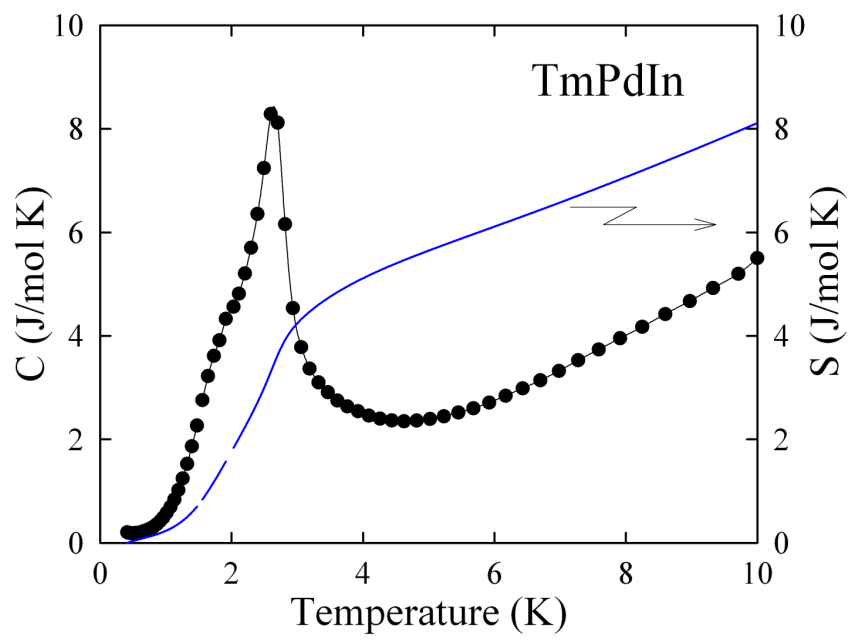

Fig. 2. Low-temperature dependence of the specific heat and the magnetic entropy (solid line) of TmPdIn.

As recalled in Table II, even smaller jump in $C(T)$ at the onset of the ordered state and strongly reduced magnetic entropy released by $T_{\mathrm{N}}=2.5 \mathrm{~K}$ were found for TmNiIn [6]. On the contrary, for TmPtIn [7] and TmAgGe [8], as well as for the compounds considered here for the first time, i.e. TmAgSn and TmAuGe, the jumps at the respective $T_{\mathrm{N} / \mathrm{C}}$ are fairly consistent with the MFA prediction. Accordingly, the entropy observed at $T_{\mathrm{N} / \mathrm{C}}$ is reasonably close to the value expected for a $\mathrm{CEF}$ doublet ground state.

TABLE II

Specific heat data of investigated TmTX compounds. $T_{\mathrm{N} / \mathrm{C}}-$ ordering temperature, $\Delta C$ - jump of the specific heat at $T_{\mathrm{N} / \mathrm{C}}, S-$ jump of the specific heat at $T_{\mathrm{N} / \mathrm{C}}$.

\begin{tabular}{c|c|c|c|c}
\hline \hline Compound & $T_{\mathrm{N}, \mathrm{C}}[\mathrm{K}]$ & $\Delta C[\mathrm{~J} / \mathrm{mol} \mathrm{K}]$ & $S[\mathrm{~J} / \mathrm{mol} \mathrm{K}]$ & Ref. \\
\hline TmNiIn & 2.5 & 3.7 & 0.9 & {$[6]$} \\
TmPdIn & 2.65 & 5.9 & 3.7 & - \\
TmPtIn & 3.5 & 12 & 4.2 & {$[7]$} \\
TmAgGe & 4.2 & 14 & 4.2 & {$[8]$} \\
TmAgSn & 4.15 & 10 & 4.0 & - \\
TmAuGe & 4.1 & 10.1 & 5.2 & -
\end{tabular}

\section{Conclusions}

All the investigated TmTX ternaries, which crystallize with the ZrNiAl-type unit cells, are antiferromagnetic 
below the Néel temperature $T_{\mathrm{N}} \leq 4.2 \mathrm{~K}$. The hexagonal LiGaGe-type structure is adopted by TmAuGe and this compound orders ferromagnetically at $T_{\mathrm{C}}=4.1 \mathrm{~K}$. In turn, TmRuGe is isostructural with the orthorhombic silicide TiNiSi and remains paramagnetic down to the lowest temperatures studied.

For all the TmTX compounds studied, the effective magnetic moment is close to the free $\mathrm{Tm}^{3+}$ ion value, whereas the moments measured in the ordered state in strong magnetic fields are distinctly smaller than the free $\mathrm{Tm}^{3+}$ ion value. This finding indicates the important role played by the CEF interactions. Moreover, it may suggest noncollinear orderings, which have indeed been revealed for TmNiIn, TmPtIn and TmAgGe by recent neutron diffraction experiments [6-8].

At low temperatures, the specific heat of the TmTX compounds is dominated by the magnetic contribution. The lambda-shaped anomalies near $T_{\mathrm{N} / \mathrm{C}}$ manifest a continuous character of the phase transitions. For TmNiIn and TmPdIn, small magnitude of the jump in the specific heat at $T_{\mathrm{N}}$ and reduced magnetic entropy released by $T_{\mathrm{N}}$ hints at induced nature of the magnetic ordering in the presence of a singlet CEF ground state. For the other ternaries investigated, the heat capacity data seems to indicate doubly degenerated electronic ground states.

\section{Acknowledgments}

The work was supported by the Ministry of Science and Higher Education (Poland) under research grant no. NN202 201039

\section{References}

[1] A. Szytuła, J. Leciejewicz, Handbook of Crystal Structures and Magnetic Properties of Rare Earth Intermetallics, CRC Press, Boca Raton, Fl. 1994.

[2] D. Gignoux, D. Schmitt, in: Handbook of Magnetic Materials, Vol. 10, Ed. K.H.J. Buschow, Elsevier Science B.V., 1997, Ch. 2, p. 239.

[3] A. Szytuła, Crystal Structures and Magnetic Properties of RTX Rare Earth Intermetallics, Wydawnictwo Uniwersytetu Jagiellońskiego, Kraków 1998.

[4] S. Baran, A. Hoser, A. Szytuła, J. Magn. Magn. Mater. 335, 97 (2013).

[5] S. Baran, J. Przewoźnik, A. Szytuła, Acta Phys. Pol. A 124, 998 (2013).

[6] S. Baran, D. Kaczorowski, A. Arulraj, B. Penc, A. Szytuła, J. Magn. Magn. Mater. 323, 833 (2011).

[7] S. Baran, D. Kaczorowski, A. Arulraj, B. Penc, A. Szytuła, J. Magn. Magn. Mater. 322, 2177 (2010).

[8] S. Baran, D. Kaczorowski, A. Arulraj, B. Penc, A. Szytuła, J. Magn. Magn. Mater. 321, 3256 (2009).

[9] J. Rodriguez-Carvajal, Physica 192, 55 (1993).

[10] N.C. Tuan, V. Sechovsky, M. Diviš, P. Svoboda, H. Nakotte, F.R. de Boer, N.H. Kim-Ngan, J. Appl. Phys. 73, 5677 (1993). 